\title{
Examen de la gestion de l'infection à la syphilis congénitale diagnostiquée avant la naissance
}

\author{
Margot Rosenthal ${ }^{1 *}$, Vanessa Poliquin ${ }^{1}$
}

\section{Résumé}

Contexte : L'incidence de la syphilis chez les Canadiennes en âge de procréer a augmenté de façon spectaculaire au cours de la dernière décennie, avec une résurgence des nourrissons nés atteints de syphilis congénitale. Même s'il existe des lignes directrices pour éclairer l'infection maternelle pendant la grossesse, il existe peu de données pour orienter la gestion dans les situations où le fœtus en développement est gravement atteint.

Examen de cas : La patiente s'est présentée au deuxième trimestre de sa grossesse après avoir contracté la syphilis. Des analyses sérologiques positives (titrage du Venereal Disease Research Laboratory [VDRL] de 1:64) et un chancre suggèrent une infection primaire. L'échographie a démontré un fœtus ayant un âge gestationnel de 19+3 semaines atteint d'hydrops fetalis et présentant un cerveau nettement anormal. L'amniocentèse a confirmé une infection congénitale à la syphilis au cours de tests de réaction en chaîne par polymérase. Après un traitement de neuf jours à la pénicilline $\mathrm{G}$ par voie intraveineuse, l'état du fœtus s'est détérioré et la famille a finalement choisi une interruption médicale de la grossesse.

Discussion : L'évolution de la technologie de l'échographie permet d'identifier les fœtus gravement touchés, qui seraient peut-être venus au monde mort-nés dans le passé. À la suite de la syphiliothérapie courante au moyen de la pénicilline benzathine, ces caractéristiques échographiques anormales peuvent prendre des semaines ou des mois à s'inverser, ce qui pose un défi au niveau du pronostic et du counseling. Les données des rapports de cas suggèrent que le traitement intensif au moyen de la pénicilline par voie intraveineuse peut être efficace dans les cas graves présentant une hydrops fetalis.

Conclusion : Ce cas met en lumière la morbidité potentielle de l'infection à la syphilis congénitale et souligne le manque de documentation actuelle. L'échange de renseignements sera essentiel pour construire une base de connaissances moderne sur le traitement de cette maladie ancienne.
Cette oeuvre est mise à la disposition selon les termes de la licence internationale Creative Commons Attribution 4.0

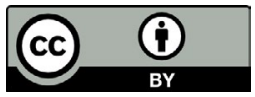

\begin{abstract}
Affiliation
1 Département d'obstétrique, de gynécologie et de sciences de la reproduction, Collège de médecine Max Rady, Université du Manitoba, Winnipeg, MB
\end{abstract}

\section{*Correspondance :}

umrose32@myumanitoba.ca

Citation proposée : Rosenthal MA, Poliquin V. Examen de la gestion de l'infection à la syphilis congénitale diagnostiquée avant la naissance. Relevé des maladies transmissibles au Canada 2022;48(2/3):125-9.

https://doi.org/10.14745/ccdr.v48i23a09f

Mots-clés : syphilis, syphilis congénitale, grossesse, résultats d'échographies, hydrops fetalis

\section{Introduction}

Les infections à la syphilis chez les Canadiennes en âge de procréer ont augmenté de façon spectaculaire au cours de la dernière décennie, avec la résurgence des grossesses touchées et des nourrissons nés atteints de syphilis congénitale (1). La transmission verticale par passage transplacentaire des spirochètes se produit chez $50 \%$ à $80 \%$ des patientes non traitées, avec les taux de transmission les plus élevés pour les infections primaires et secondaires (2). Les grossesses touchées présentent un risque accru de mortinaissance et d'accouchement prématuré (3). Le traitement prénatal au moyen de la pénicilline benzathine $\mathrm{G}$ par voie intramusculaire est très efficace pour prévenir la syphilis congénitale (4). Dans une minorité de cas, les séquelles de l'infection fœtale peuvent être détectées par échographie (5). Il y a un manque de données probantes disponibles pour orienter les cliniciens qui s'occupent de ces grossesses touchées. 


\section{TÉMOIGNAGE}

\section{Présentation de cas}

Il s'agit du cas d'une femme G4P3 de 29 ans

(quatrième grossesse; trois accouchements) qui a reçu des soins dans une communauté rurale au Manitoba, au Canada, puisqu'elle a été en contact avec la syphilis. Elle s'est présentée enceinte sans être sûre de la date de ses dernières menstruations et ses antécédents médicaux et chirurgicaux étaient non contributoire. Étant donné son risque en tant que contact sexuel, elle a été traitée pour la syphilis de façon empirique avec une dose de pénicilline benzathine $G$ par voie intramusculaire. Elle s'est plainte de douleurs dorsales et de faiblesses qui se sont aggravées pendant plusieurs semaines avec une difficulté croissante à bouger le cou, ce qui a entraîné le transfert à notre point de soins tertiaires pour une évaluation et une gestion plus poussées.

Une lésion vulvaire non douloureuse et peu profonde a été détectée à la fourchette postérieure, qui a fait l'objet d'un prélèvement par écouvillonnage et qui s'est finalement révélée positive pour l'acide désoxyribonucléique de Treponema pallidum ( $T$. pallidum) au cours des tests de réaction en chaîne par polymérase (PCR). Ses symptômes cliniques ont soulevé des préoccupations pour la neurosyphilis puisqu'elle a obtenu un résultat positif à un test de Jolt suggérant une irritation méningée même si les signes de Brudzinsky et de Kernig sont négatifs. La force dans les extrémités inférieures était objectivement réduite. Elle n'avait pas de fièvre ni d'éruption cutanée.

Elle a été admise à l'unité antépartum du Health Science Center de Winnipeg et cogérée par les services des maladies infectieuses, des maladies infectieuses reproductives et de la médecine fœtale et maternelle. Une ponction lombaire a été effectuée et un traitement antibiotique a commencé au moyen de la pénicilline $G$ par voie intraveineuse (24 millions d'unités par jour divisées toutes les quatre heures conformément aux recommandations de dose pour la neurosyphilis). Le liquide céphalorachidien présentait de faibles niveaux de protéines $(0,12 \mathrm{~g} / \mathrm{L})$ et des niveaux de glucose normaux, avec un nombre total normal de cellules nucléées de $1 \times 10^{6} / \mathrm{L}$. La tomographie par ordinateur sans perfusion et l'imagerie par résonance magnétique du cerveau et de la colonne vertébrale (effectuées sans gadolinium parce qu'il est contre-indiqué pendant la grossesse) n'ont pas démontré d'anomalies aiguës. Elle était atteinte d'anémie microcytaire avec un niveau d'hémoglobine de $77 \mathrm{~g} / \mathrm{L}$ et de marqueurs inflammatoires avec un taux de sédimentation érythrocytaire supérieur à $140 \mathrm{~mm} /$ heure et un niveau de protéine $\mathrm{C}$-réactive de $58 \mathrm{mg} / \mathrm{L}$.

L'infection syphilitique maternelle a été confirmée au moyen du titrage sérique du Venereal Disease Research Laboratory (VDRL) de 1:64 et les lgG de T. pallidum ont été détectés par test d'immunologie des microparticules chimioluminescentes. En plus du résultat positif du test PCR pour la lésion vulvaire confirmant un chancre, un examen sérologique de l'année précédente a confirmé qu'il s'agissait d'une infection primaire. Aucune autre infection sexuellement transmissible n'a été détectée et des résultats sérologiques supplémentaires sont présentés au tableau 1. L'analyse du liquide céphalorachidien pour le VDRL était négative et toutes les cultures (bactériennes, mycobactériennes et fongiques) étaient négatives.

Tableau 1 : Examens de laboratoire chez la mère

\begin{tabular}{|c|c|c|}
\hline Test & Source & Résultat \\
\hline $\begin{array}{l}\text { Test Ab IgG+lgM de } \\
\text { Treponema pallidum }\end{array}$ & Sérum & Positif \\
\hline VDRL & Sérum & Réactif à 1:64 \\
\hline $\begin{array}{l}\text { IgM/lgG de Toxoplasma } \\
\text { gondii }\end{array}$ & Sérum & Négatif/négatif \\
\hline $\begin{array}{l}\text { lgM/lgG du } \\
\text { cytomégalovirus }\end{array}$ & Sérum & Négatif/anticorps détecté \\
\hline $\begin{array}{l}\operatorname{lgM} / \operatorname{lgG} \text { du parvovirus } \\
\text { B19 }\end{array}$ & Sérum & Négatif/indéterminé \\
\hline $\begin{array}{l}\text { IgM du virus du Nil } \\
\text { occidental }\end{array}$ & Sérum & Négatif \\
\hline $\begin{array}{l}\text { IgG d'Anaplasma } \\
\text { phagocytophilum }\end{array}$ & Sérum & Négatif \\
\hline $\begin{array}{l}\text { Test PCR/TAAN pour } \\
\text { I'ADN de } \\
\text { Treponema pallidum }\end{array}$ & $\begin{array}{l}\text { Lésion } \\
\text { vulvaire }\end{array}$ & Cible détectée \\
\hline VDRL du LCR & LCR & Non réactif \\
\hline $\begin{array}{l}\text { Test PCR/TAAN pour } \\
\text { I'ADN de } \\
\text { Treponema pallidum }\end{array}$ & LCR & Cible non détectée \\
\hline Culture bactérienne & LCR & Négatif \\
\hline Culture mycobactérienne & LCR & Négatif \\
\hline Culture fongique & LCR & Négatif \\
\hline
\end{tabular}

Abréviations : $L C R$, liquide céphalorachidien; $\operatorname{lgM} / \mathrm{lgG}$, immunoglobuline $\mathrm{M} / \mathrm{immunoglobuline} \mathrm{G}$; PCR ADN/TAAN, réaction en chaîne par polymérase de l'acide désoxyribonucléique/test d amplification des acides nucleiques; VDRL, Venereal Disease Research Laboratory

L'échographie réalisée en médecine fœtale-maternelle a démontré une grossesse intra-utérine vivante à l'âge gestationnel de 19+3 semaines. Le fœtus a été affecté par l'hydrops fetalis grave, comme en témoignent les grandes effusions pleurales, de grandes ascites qui compressent les reins et l'œdème de la paroi corporelle (figure 1). Le cerveau était très anormal avec un liquide céphalo-rachidien clair et des échos rayés dans tout le cortex (figure 2). Il y avait des polyhydramnios avec une poche verticale maximale de $8 \mathrm{~cm}$, accompagné d'un placenta épais et ouvertement hydropique (figure 3). L'échographie Doppler de l'artère ombilicale était anormale avec un rapport systoliquediastolique accru. Cependant, le Doppler de l'artère cérébrale moyenne n'a pas démontré d'anémie fœtale (vitesse systolique de pointe 1,37 MoM). Pour effectuer l'évaluation de l'hydrops fetalis, une amniocentèse a été effectuée et elle a été envoyée pour une évaluation microbiologique et génétique. Les résultats sont résumés dans le tableau 2 et le test PCR était entre autres positif pour T. pallidum. 
Figure 1 : De très grandes ascites, des intestins entassés et des reins comprimés suggérant une pression intra-abdominale élevée à l'âge gestationnel de $19+3$ semaines

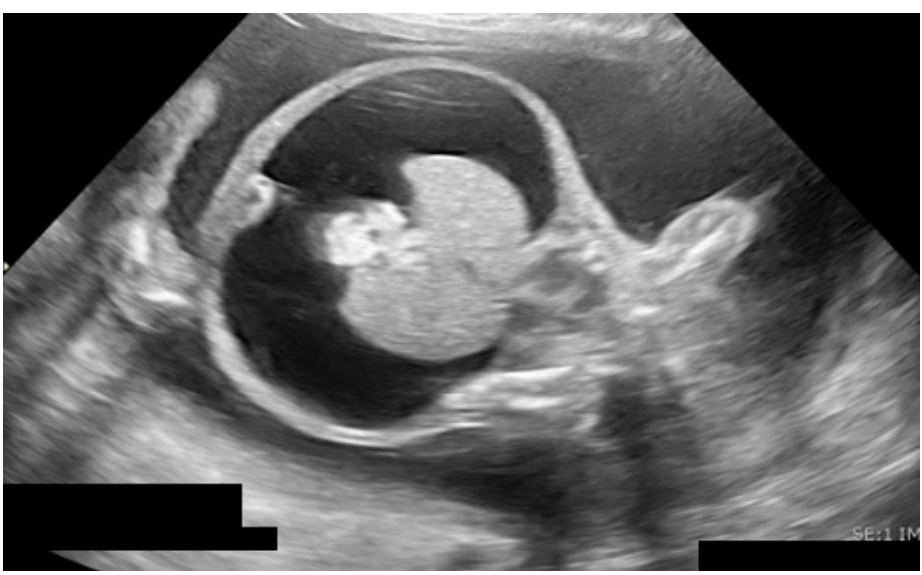

Figure 2 : Gdème marqué au cuir chevelu, cerveau très anormal avec un liquide céphalo-rachidien clair, échos rayés dans toute la matière cérébrale à l'âge gestationnel de 19+3 semaines

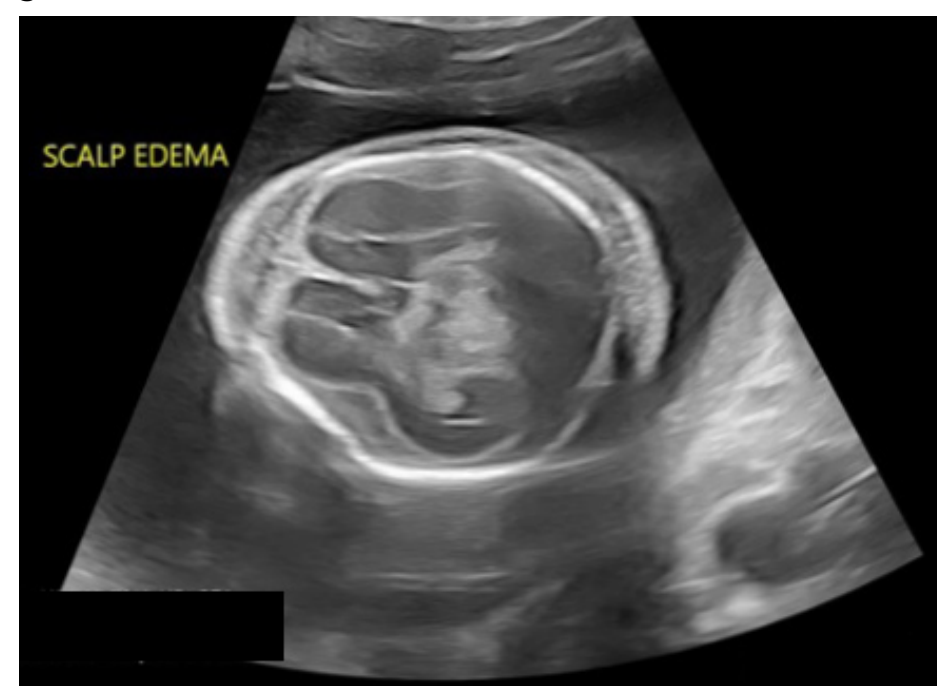

Figure en anglais seulement

Le traitement se poursuit au moyen de la pénicilline par voie intraveineuse dans l'espoir d'atteindre des niveaux suffisants pour traiter l'infection fœtale. Après neuf jours, une échographie détaillée répétée a démontré une aggravation de l'ascite fœtale. Le fœtus avait maintenant un âge gestationnel de $20+5$ semaines avec une circonférence abdominale de $282 \mathrm{~mm}$ (taille à 32+2 semaines) (figure 4). L'œdème du cuir chevelu et les résultats de l'échographie Doppler sont demeurés stables. La famille a reçu des conseils détaillés sur les options, y compris l'interruption de grossesse ou la gestion de grossesse à l'aide d'une série d'échographies et d'imageries par résonance magnétique fœtale pour suivre les changements neurologiques structuraux. Dans l'ensemble, le pronostic était jugé médiocre
Figure 3 : Placenta hydropique et élargi avec de multiples lésions kystiques à l'âge gestationnel de $19+3$ semaines

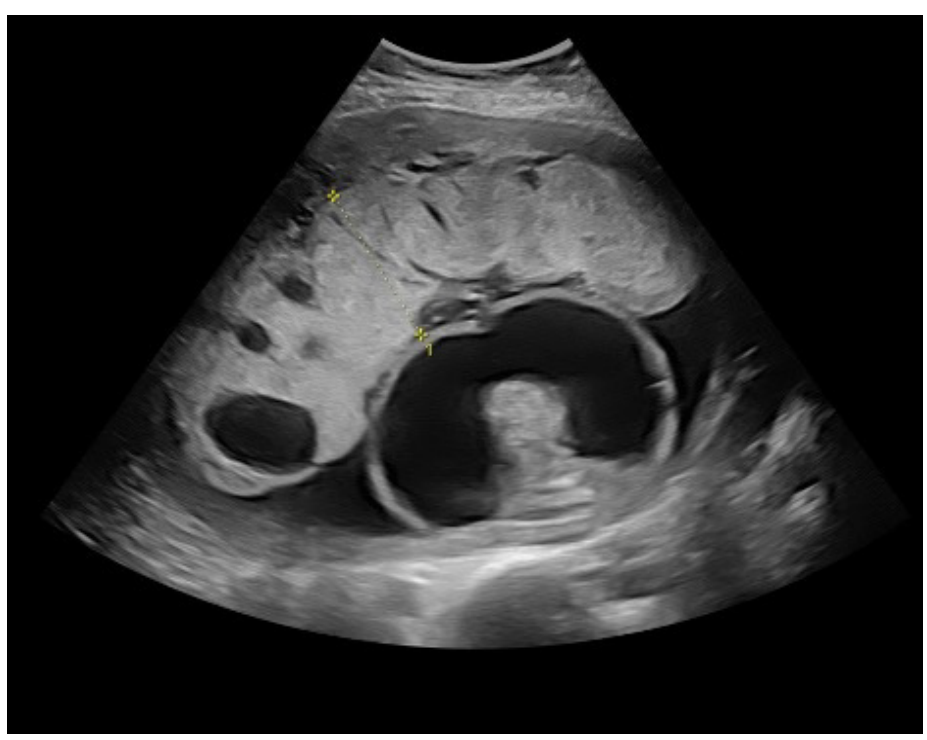

Tableau 2 : Résultats de l'amniocentèse

\begin{tabular}{|l|l|l|}
\multicolumn{1}{|c|}{ Test } & \multicolumn{1}{c|}{ Source } & \multicolumn{1}{c|}{ Résultat } \\
\hline $\begin{array}{l}\text { Test PCR/TAAN pour } \\
\text { l'ADN de } \\
\text { Treponema pallidum }\end{array}$ & $\begin{array}{l}\text { Liquide } \\
\text { amniotique }\end{array}$ & Cible détectée \\
\hline $\begin{array}{l}\text { TAAN de l'ADN du } \\
\text { cytomégalovirus }\end{array}$ & $\begin{array}{l}\text { Liquide } \\
\text { amniotique }\end{array}$ & Cible non détectée \\
\hline $\begin{array}{l}\text { TAAN de l'ADN du } \\
\text { parvovirus B19 }\end{array}$ & $\begin{array}{l}\text { Liquide } \\
\text { amniotique }\end{array}$ & Cible non détectée \\
\hline Culture virale & $\begin{array}{l}\text { Liquide } \\
\text { amniotique }\end{array}$ & Négatif \\
\hline
\end{tabular}

Abréviations : PCR ADN/TAAN, réaction en chaîne par polymérase de l'acide désoxyribonucléique/test d'amplification des acides nucléiques; TAAN de l'ADN, test d'amplification des acides nucléiques de l'acide désoxyribonucléique

compte tenu de l'étendue de l'hydrops fetalis au début de la gestation et de la gravité des changements dans le cortex cérébral. Ultimement, la famille a décidé de mettre fin à la grossesse. L'induction médicale du travail a été effectuée avec la mifépristone suivie du misoprostol par voie vaginale. Un nourrisson hydropique mort-né de sexe féminin a été mis au monde révélant une mort intrapartum, à un âge gestationnel de 21+0 semaines et pesant $747 \mathrm{~g}$ (plus du $99^{\mathrm{e}}$ centile pour l'âge gestationnel). La famille a refusé une autopsie. Le placenta pesait $387 \mathrm{~g}$ (plus de deux fois le poids moyen pour cet âge gestationnel) avec des signes pathologiques d'hémorragie déciduale, de nécrose et d'inflammation aiguë.

Les symptômes maternels ont continué de s'améliorer après l'accouchement et l'arrêt de la pénicilline par voie intraveineuse. Une fois en mesure de se déplacer sans assistance, elle a été renvoyée chez elle. La sérologie de suivi a montré une réponse de traitement appropriée avec son titrage VDRL à 1:4. 


\section{Figure 4 : Aggravation des ascites avec un abdomen ayant la taille de celui d'un fœtus ayant un âge gestationnel de 32 semaines à un âge gestationnel de $20+5$ semaines}

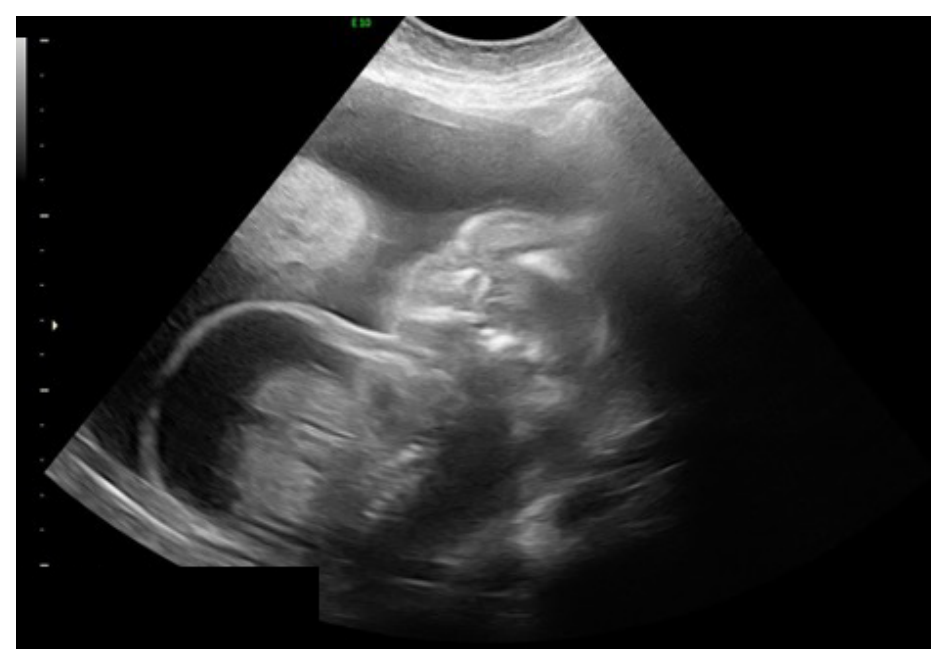

\section{Discussion}

La syphilis congénitale est une maladie évitable. Dans un énoncé de 2019, la Société des obstétriciens et gynécologues du Canada a plaidé pour un dépistage adéquat pendant la grossesse, avec un dépistage amélioré en trois points dans les zones d'éclosion (1). Il a été démontré que le traitement de la syphilis pendant la grossesse est très efficace pour réduire les taux de syphilis congénitale, de mortinaissance syphilitique et de complications obstétriques comme la naissance prématurée (3). Le traitement de choix est la pénicilline benzathine $G$ par voie intramusculaire à une dose de 2,4 millions d'unités, avec une deuxième dose administrée une semaine plus tard (3). Une troisième dose est recommandée pour les patientes atteintes de syphilis latente tardive (3).

Compte tenu du risque accru de restriction de la croissance intra-utérine, d'anomalies fœtales et d'hydrops fetalis, une surveillance sonographique supplémentaire est recommandée (6). Dans une étude de cohorte sur les femmes séropositives qui ont subi une échographie avant le traitement de la syphilis, Rac et al. ont constaté que $30 \%$ présentaient des signes de syphilis congénitale (5). L'hépatomégalie fœtale était l'anomalie la plus remarquée (observée chez $79 \%$ des fœtus touchés) et la placentomégalie, le polyhydramnios, les ascites et les données probantes d'anémie fœtale avec une vitesse systolique de pointe élevée au cours de l'échographie Doppler de l'artère cérébrale moyenne ont également été observées (5). Les auteurs ont suivi la chronologie de la résolution des anomalies à l'échographie de Doppler et ont trouvé que les anomalies à l'échographie de Doppler de l'artère cérébrale moyenne les ascites et le polyhydramnios étaient les premiers signes à se résoudre (résolution de $80 \%$ dans les 40 jours), suivis par la placentomégalie et l'hépatomégalie, qui pourraient persister jusqu'à l'accouchement. Il est à noter que les résultats par échographie de la syphilis congénitale ne sont pas couramment détectés avant la gestation de 18 semaines, car le système immunitaire fœtal immature n'est pas encore en mesure de produire une réponse suffisante $(3,6)$.

Historiquement, la syphilis congénitale était diagnostiquée après la naissance, la plupart des nouveau-nés infectés présentant des symptômes avant le deuxième mois de vie (6). Les technologies modernes, y compris l'échographie, ont démontré jouer un rôle en évolution dans le diagnostic de la syphilis congénitale au cours des trois dernières décennies. Une grande partie du savoir médical dans le traitement de la syphilis est antérieure à ces diagnostics prénatals, laissant le clinicien moderne avec un diagnostic, mais des données probantes limitées pour orienter le traitement.

Dans le cas de la patiente, le traitement a été amorcé à l'aide de pénicilline $G$ par voie intraveineuse selon le schéma posologique de la neurosyphilis pour l'indication maternelle. Le traitement se poursuit dans l'espoir de traiter l'infection fœtale. Un rapport de cas de Galan et al. décrit l'utilisation de la pénicilline $G$ par voie intraveineuse pour traiter l'hydrops fetalis liée à la syphilis à 24 semaines de gestation après qu'une échographie a montré des caractéristiques d'aggravation 10 jours après l'administration de la pénicilline benzathine par voie intramusculaire (7). Leur patiente a présenté une résolution complète concernant les résultats sonographiques après 10 jours de thérapie par voie intraveineuse (7). Un deuxième cas décrit par Chen et al. a fait état d'une patiente ayant reçu un diagnostic de syphilis secondaire à 28 semaines de grossesse avec des résultats sonographiques d'hydrops fetalis et d'anémie fœtale, et avec une vitesse systolique de pointe élevée de l'artère cérébrale moyenne (8). Leur patiente a été admise et traitée au moyen de la pénicilline $G$ pendant 14 jours afin de cibler des niveaux fœtaux maximaux et soutenus (8). La patiente a subi une cordocentèse guidée par échographie et une transfusion fœtale, et a présenté la résolution de l'hydrops fetalis en deux semaines (8). Même si les deux cas ont connu une résolution relativement rapide des symptômes comparativement aux données de la cohorte de Rac, il n'y a pas assez de renseignements pour tirer des conclusions sur le traitement optimal pour la syphilis fœtale.

Le premier cas décrit une situation qui, sans traitement, aurait presque certainement progressé vers la mortinaissance syphilitique. Les directives actuelles en matière d'obstétrique pour les enquêtes sur la mortinaissance sont vagues en matière de recommandations relatives au dépistage des infections, de nombreux hôpitaux locaux élaborant leurs propres protocoles (9). Cela mène à la possibilité de diagnostics ambigus de syphilis pendant l'enquête sur l'hydrops fetalis non immunitaire et la mortinaissance, avec des conséquences pour les deux patientes et une meilleure santé publique. 


\section{Conclusion et appel à l'action}

Ce cas met en évidence la gravité potentielle de l'infection à la syphilis congénitale et souligne la rareté de la documentation actuelle pour guider la gestion d'un fœtus gravement touché au cours de la période antépartum. L'échange de renseignements sera essentiel pour construire une base de connaissances moderne sur le traitement de cette maladie résurgente. On exhorte les fournisseurs de soins obstétricaux à augmenter le dépistage infectieux et à maintenir la syphilis comme diagnostic différentiel lorsqu'ils enquêtent sur des complications graves.

\section{Déclaration des autrices}

Les autrices n'ont aucun conflit d'intérêts à divulguer.

Le contenu de l'article et les points de vue qui y sont exprimés n'engagent que les auteurs et ne correspondent pas nécessairement à ceux du gouvernement du Canada.

\section{Intérêts concurrents}

Aucun.

\section{Remerciements}

Les autrices souhaitent remercier la Dre C Schneider pour sa contribution à ce cas.

\section{Financement}

Cette publication n'a pas été financée.

\section{Références}

1. The Society of Obstetricians and Gynaecologists of Canada. SOGC Infectious Diseases Committee Statement on Syphilis. Ottawa (ON): SOGC; 2019. https://sogc.org/en/ content/featured-news/sogc-infectious-diseases-committeestatement-on-syphilis.aspx

2. Gomez GB, Kamb ML, Newman LM, Mark J, Broutet N, Hawkes SJ. Untreated maternal syphilis and adverse outcomes of pregnancy: a systematic review and meta-analysis. Bull World Health Organ 2013;91(3):217-26. DOI PubMed

3. Adhikari EH. Syphilis in Pregnancy. Obstet Gynecol 2020;135(5):1121-35. DOI PubMed

4. Alexander JM, Sheffield JS, Sanchez PJ, Mayfield J, Wendel GD Jr. Efficacy of treatment for syphilis in pregnancy. Obstet Gynecol 1999;93(1):5-8. DOI PubMed

5. Rac MW, Bryant SN, Mclntire DD, Cantey JB, Twickler DM, Wendel GD Jr, Sheffield JS. Progression of ultrasound findings of fetal syphilis after maternal treatment. Am J Obstet Gynecol 2014;211(4):426.e1-6. DOI PubMed

6. Rac MW, Stafford IA, Eppes CS. Congenital syphilis: A contemporary update on an ancient disease. Prenat Diagn 2020;40(13):1703-14. DOI PubMed

7. Galan HL, Yandell PM, Knight AB. Intravenous penicillin for antenatal syphilotherapy. Infect Dis Obstet Gynecol 1993;1(1):7-11. DOI PubMed

8. Chen I, Chandra S, Singh A, Kumar M, Jain V, Turnell R. Successful outcome with intrauterine transfusion in non-immune hydrops fetalis secondary to congenital syphilis. J Obstet Gynaecol Can 2010;32(9):861-5. DOI PubMed

9. Leduc L. No. 394-stillbirth Investigation. J Obstet Gynaecol Can 2020;42(1):92-9. DOI PubMed 